\title{
Nucleotide metabolism pathway: the achilles' heel for bacterial pathogens
}

\author{
Sujata Kumari ${ }^{1,2, *}$ and Prajna Tripathi ${ }^{1,3}$ \\ ${ }^{1}$ National Institute of Immunology, New Delhi 110 067, India \\ ${ }^{2}$ Present address: Department of Zoology, Magadh Mahila College, Patna University, Patna 800001 , India \\ ${ }^{3}$ Present address: Institute of Molecular Medicine, Jamia Hamdard, New Delhi 110 062, India
}

Pathogens exploit their host to extract nutrients for their survival. They occupy a diverse range of host niches during infection which offer variable nutrients accessibility. To cause a successful infection a pathogen must be able to acquire these nutrients from the host as well as be able to synthesize the nutrients on its own, if required. Nucleotides are the essential metabolite for a pathogen and also affect the pathophysiology of infection. This article focuses on the role of nucleotide metabolism of pathogens during infection in a host. Nucleotide metabolism and disease pathogenesis are closely related in various pathogens. Nucleotides, purines and pyrimidines, are biosynthesized by the de novo and salvage pathways. Whether the pathogen will employ the de novo or salvage pathway during infection is dependent on various factors, like availability of nucleotides, energy condition and presence of enzymes of the particular pathway. Understanding the nucleotide metabolism of a pathogen within its host will provide a key insight into the hostpathogen interaction and will also aid in the development of novel therapeutic strategies.

Keywords: Drug target, host niches, nucleotide biosynthesis, pathogens, virulence.

NUCLEOTIDES are essential metabolites for all living organisms. They play a vital role in all biological aspects of the cell. Most importantly, they are the building blocks for synthesis of DNA and RNA, and hence are involved in replication and transcription processes. Nucleotides are also the constituents of essential coenzymes like NAD and $\mathrm{FAD}^{1}$. They are also known to activate the precursors involved in lipid and carbohydrate synthesis ${ }^{2,3}$ and are the sole energy currency of the cell. Further, cyclic derivatives of purine nucleotides, cAMP and cGMP, serve as intracellular second messengers in numerous signalling pathways ${ }^{4}$.

\section{Nucleotide biosynthesis pathway}

Nucleotides, purines and pyrimidines, are biosynthesized in the cell by the de novo or salvage pathway. In the

*For correspondence. (e-mail: sujatakumari@nii.ac.in) de novo pathway, the nucleotides are synthesized from simple precursor molecules. In the salvage pathway, the preformed nucleobases or nucleosides which are present in the cell or transported from external environmental milieu to the cell are utilized to form nucleotides.

\section{Purine biosynthesis pathway}

The purine biosynthesis pathway is universally conserved in living organisms (Figure 1). As an example, we here present the pathway derived from well-studied Grampositive bacteria Lactococcus lactis. In the de novo pathway the purine nucleotides are synthesized from simple molecules such as phosphoribosyl pyrophosphate (PRPP), amino acids, $\mathrm{CO}_{2}$ and $\mathrm{NH}_{3}$ by a series of enzymatic reactions. The first ten reactions lead to the synthesis of inosine monophosphate (IMP). Further, adenosine monophosphate is synthesized from IMP by the action of adenylosuccinate synthase (purA) and adenylosuccinate lyase (purB), while guanosine monophosphate is produced from IMP by the help of enzyme IMP dehydrogenase $(g u a B)$ and guanosine monophosphate synthase (guaA). The enzymes of this pathway are highly regulated. The first step of the pathway is a rate-limiting step catalysed by the enzyme encoded by phosphoribosyl pyrophosphate amidotransferase ( $p u r F)$.

Purines are synthesized in the salvage pathway from nucleobases and nucleosides which are either present inside the cell or transported into the cell by various nucleobase and nucleoside transporters. Purine nucleoside phosphorylases catalyse the conversion of purine nucleosides into their corresponding nucleobase and ribose-1-phosphate. Purine nucleobases are converted into nucleoside monophosphate by the activity of nucleobase phosphoribosyl transferases. Nucleoside kinases catalyse the conversion of nucleoside monophosphate into nucleoside di- or triphosphate.

\section{Pyrimidine biosynthesis pathway}

Like the purine biosynthesis pathway, the pyrimidine biosynthesis pathway is also conserved across all organisms (Figure 2). The pathway described here is again derived 


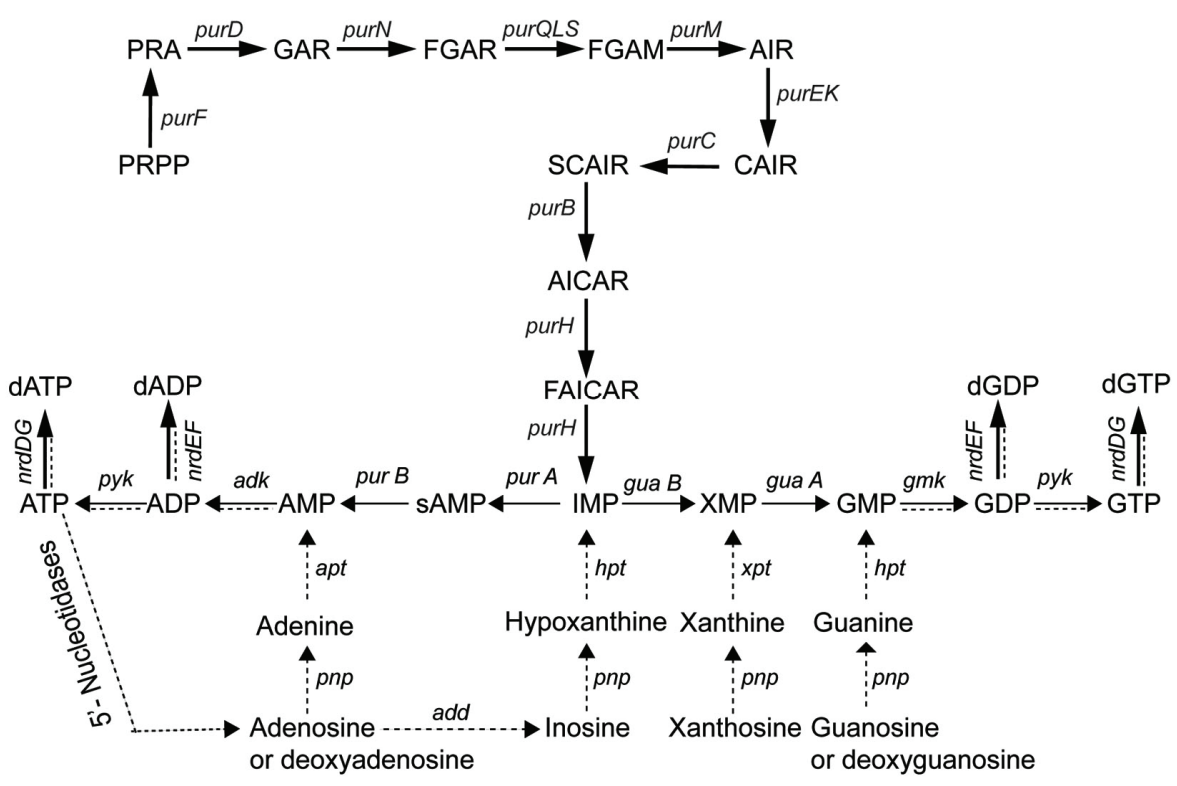

Figure 1. Purine biosynthesis by de novo and salvage pathways. The de novo pathway is denoted by solid arrows, while the salvage pathway is denoted by dashed arrows. Reactions common to them are denoted by solid and dashed arrows. The genes encoding the enzymes involved are indicated. PRPP, Phosphoribosyl pyrophosphate; PRA, Phosphoribosyl amine; GAR, Glycinamide ribonucleotide; FGAR, Formylglycinamide ribonucleotide; FGAM, Formylglycinamidine ribonucleotide; AIR, Aminoimidazole ribonucleotide; CAIR, Phosphoribosyl carboxyaminoimidazole; SAICAR, Succinocarboxyamide carboxyaminoimidazole ribonucleotide; AICAR, Aminoimidazole carboxamide ribonucleotide; FAICAR, Formaminoimidazole carboxamide ribonucleotide; IMP, Inosine monophosphate; XMP, Xanthosine monophosphate; GMP, Guanosine monophosphate; GDP, Guanosine diphosphate; GTP, Guanosine triphosphate; dGDP, Deoxyguanosine diphosphate; dGTP, Deoxyguanosine triphosphate; sAMP, Adenylsuccinate; AMP, Adenosine monophosphate; ADP, Adenosine diphosphate; ATP, Adenosine triphosphate; dADP, Deoxyadenosine diphosphate; dATP, Eoxyadenosine triphosphate. pur $F$, PRPP amidotransferase; purD, GAR synthase; purN, GAR transformylase; purQLS, FGAM synthase; purM, AIR synthase; purEK, CAIR synthase; $p u r C$, SAICAR synthase; $p u r B$, Adenylosuccinate lyase; $p u r H$, IMP cyclohydolase; guaB, IMP dehydrogenase; guaA, GMP synthase; gmk, Guanylate kinase; $p y k$, Pyruvate kinase; $n r d E F$, Aerobic ribonucleotide diphosphate reductase; $n r d D G$, Anaerobic riobonucleotide triphosphate reductase; purA, Adenylosuccinate synthase; purB, Adenylosuccinate lyase; adk, Adenylate kinase; pnp, Purine nucleoside phosphorylase; add, Adenosine deaminase; apt, Adenine phosphoribosyltransferse; $h p t$, Hypoxanthine/guanine phosphoribosyltransferase and $x p t$, Xanthine phosphoribosyltransferse.

from L. lactis. The de novo synthesis of the pyrimidine nucleoside triphosphates, uridine monophosphate (UTP) and cytidine triphosphate (CTP), occurs in a linear pathway initiated by the formation of carbamoyl phosphate from bicarbonate, glutamine and ATP. The pyrimidine base orotate is produced from carbamoyl phosphate in three steps and a phosphoribosyl group is subsequently attached to orotate resulting in the formation of pyrimidine nucleotide orotidine $5^{\prime}$-monophosphate (OMP). Uridine monophosphate (UMP) is formed by decarboxylation of OMP and converted to UTP by kinase reactions. Lastly, CTP is produced from UTP by replacing the 4'-OH group with an amino group. The enzymes involved in this pathway are tightly regulated. CTP synthase, catalysing the formation of CTP from UTP, is regulated allosterically by guanosine triphosphate (GTP $)^{5}$.

The salvage pathway uses pyrimidine bases and nucleosides to synthesize pyrimidine nucleotides. The pyrimidine bases or nucleosides are produced either by nucleotide degradation inside the cell or imported from external environment into the cell by permeases. Uridine, cytidine and thymidine are converted to their nucleobase form, i.e. uracil, cytosine and thymine respectively, by pyrimidine nucleoside phosphorylases. Uracil is converted to UMP by the enzyme uracil phosphoribosytransferase. Cytosine is deaminated to uracil by the enzyme cytosine deaminase. The uracil thus produced is also converted to UMP. The UMP produced by uracil and cytosine is converted to UDP, UTP and CTP, as discussed earlier for de novo nucleotide biosynthesis.

\section{De novo or salvage: which one to rely on during infection}

It is still an intriguing question about the utilization of de novo or salvage pathway during the infection process. The de novo pathway synthesizes nucleotides on its own and hence it may be required to sustain in the niches that have very low nucleotide availability. On the other hand, the salvage pathway is energy-efficient, unlike the de novo pathway and hence may be favourable in conditions of low energy availability or rapid multiplication for maintaining the nucleotide pool. Some pathogens, like unicellular parasites, lack the de novo pathway and rely completely 


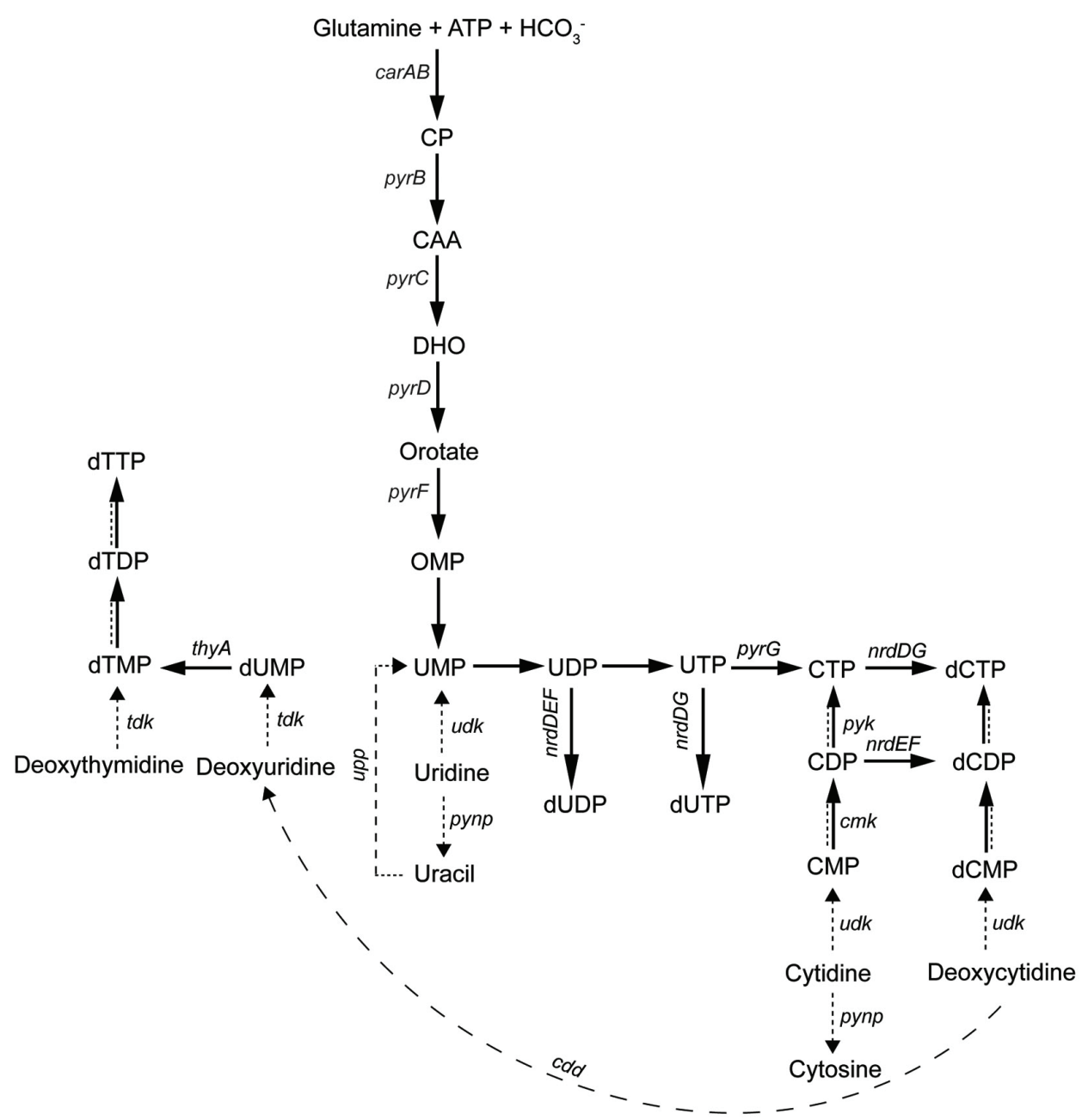

Figure 2. Pyrimidine biosynthesis pathway - de novo and salvage pathway. The solid arrows represent the de novo pathway and dashed arrows represent salvage pathway. The enzymes catalysing the reactions are denoted by their gene names. CP, Carbamoyl phosphate; CAA, Carbamoylaspartate; DHO, Dihydroorotate; OMP, Orotate monophosphate; UMP, Uridine monophosphate; UDP, Uridine monophosphate; UTP, Uridine monophosphate; dUMP, Deoxyuridine monophosphate; dUDP, Deoxyuridine monophopsphate; dUTP, Deoxyuridine triphosphate; CMP, Cytidine monophosphate; CDP, Cytidine diphosphate; CTP, Cytidine triphosphate; dCMP, Deoxycytidine monophosphate; dCDP, Deoxycytidine diphosphate; dCTP, Deoxycytidine triphosphate; dTMP, Thymidine monophosphate; dTDP, Thymidine diphosphate; dTTP, Thymidine triphosphate. carAB, Carbamoylphosphate; pyrB, Aspartate transcarbamoylase; pyrC, Dihydroorotase; pyrD, Dihydroorotate dehydrogenase; $p y r E$, Orotate phosphoribosyltransferase; $p y r F$, OMP decarboxylase; $p y r G$, CTP synthase; $c m k$, CMP kinase; $u d k$, Uridine kinase; upp, Uracil phosphoribosyltranferase; pynp, Pyrimidine nucleoside phosphorylase; $t d k$, Thymidine kinase and thy $A$, Thymidylate synthase.

on the salvage pathway for their nucleotide requirement. Next we discuss in detail the role of nucleotide de novo and salvage pathways during infection.

\section{Role of de novo pathway of nucleotides in bacterial physiology and virulence}

There are a plethora of evidences which describe the role of the de novo biosynthesis pathway of purines and pyrimidines for bacterial virulence. Escherichia coli lacking the genes involved in de novo purine and pyrimidine biosynthesis is impaired in growth in the human serum. A library of 3985 single-gene deletion mutants of non- essential genes of $E$. coli have been screened for their ability to grow in blood. Twenty-two mutants were identified which had growth defect in the human serum. Among these, 15 were deficient in genes involved in de novo purine and pyrimidine biosynthesis pathway, demonstrating that $E$. coli needs to synthesize nucleotides to survive in the human serum. Salmonella Typhimurium mutants defective in purine and pyrimidine biosynthesis have 10-100-fold reduction in viable cell count compared to wildtype strain when grown in the human serum. Similar results were obtained in Bacillus anthracis, where purE and purK (purine biosynthesis) and carA and pyrC (pyrimidine biosynthesis) deletion mutants had 5-1000-fold 
reduction in viable bacterial count compared to wildtype strain. In the mice model of anthrax infection, the purE mutants of $B$. anthracis were avirulent and mice infected with mutant strains survived till the end of the experiment, while the purK mutants were as virulent as the wildtype strain $^{6}$. In another study ${ }^{7}$, purine biosynthesis genes were found to be essential for Staphylococcus aureus pathogenicity and growth in human blood. De novo biosynthesis genes purA and purB are required for growth on human blood. The purA mutant requires adenine for its growth, while the purB mutant requires adenine as well as guanine for its growth. pur $A$ and $p u r B$ mutants are severely attenuated in virulence in zebrafish embryo model of systemic infection, which is not strainspecific. With the help of bacterial load experiments, these mutants have been shown to be incapable of replication in zebrafish embryo. Another interesting finding of this study ${ }^{7}$ was the essentiality of the $p a b A$ gene for virulence of $S$. aureus. pabA is involved in tetrahydrofolate (THF) biosynthesis. THF and pyrimidine salvage pathway are involved in the synthesis of deoxythymidine monophosphate (dTMP). Deletion of pabA leads to scarcity of dTMP and hence growth inhibition. Thymidine when supplemented exogenously in the growth medium supports the growth of pabA mutant ${ }^{7}$. purL encoding phosphoribosylformyl glycinamidine synthase that catalyses the fourth step of purine de novo biosynthesis was identified as $S$. aureus virulence gene in murine model of bacteraemia ${ }^{8}$. Augmented golden pigment production was observed in $S$. aureus strains carrying mutation in the genes involved in purine biosynthesis pathway, namely purA, purN, purH and purD. Golden pigment shields $S$. aureus from oxidative damage by host immune response ${ }^{9}$. Using microarray analysis, the authors had further shown that purine biosynthesis affects the expression of around 400 genes involved in a wide range of functions, including virulence 9 . In murine abscess model of infection, purH mutant was severely attenuated in growth in vivo. Mice infected with purH mutant strain displayed 5-log reduction in bacterial loads in the liver and kidney compared with mice infected with wildtype Newman strain. purA mutant strain also showed reduced survival in the kidney of mice compared to their wildtye counterpart. Methicillin-resistant $S$. aureus (MRSA) persistent infection outcome is dependent on purine biosynthesis. Greater expression (more than two-fold) of de novo purine biosynthesis genes purN, purM, purF, purH and purL was observed in MRSA strains causing persistent bacteraemia $(\mathrm{PB})$ compared to MRSA strains causing resolving bacteraemia $(\mathrm{RB})$. Inhibition of purine biosynthesis either by inhibitors like 6-thioguanine and mycophenolic acid, or by genetic disruption in MRSA strains causing PB converted them to MRSA strains RB-like phenotype $^{10}$. Guanine biosynthesis by de novo pathway is essential for $S$. aureus growth in the human serum. guaA or guaB (enzymes involved in guanine de novo biosyn- thesis) deletion mutants of $S$. aureus are guanine auxotroph and avirulent in mice model of infection ${ }^{11}$. Thymidine-dependent small colony variants (TD-SCVs) of $S$. aureus are commonly recovered from patients with chronic infections. TD-SCVs has several mutation in thymidylate synthetase $($ thy $A)$, a gene in the pyrimidine biosynthesis pathway. Deletion of thy A resulted in compromised virulence of $S$. aureus in mice model of infection and in Caenorhabditis elegans virulence assay. Microarray analysis indicated that various virulence gene regulators were downregulated, and the genes involved in purine and pyrimidine biosynthesis were significantly affected in thy $A$ deletion strain compared to wildtype ${ }^{12}$. purH which catalyses the last step of synthesis of IMP in purine de novo biosynthesis is shown to be crucial for virulence of $B$. anthracis in guinea pigs, irrespective of the route of infection. Interestingly, in the mice model of infection, the route of infection determined the disease outcome. purH mutant was as virulent as wildtype in intranasal infection model, while in intraperitoneal route of infection the purH mutant was partially attenuated in virulence $^{13}$. Salmonella Typhimurium and Salmonella dublin become purine auxotroph after mutation in de novo purine biosynthesis genes. Salmonella Typhimurium and $S$. dublin purA mutants were avirulent, whereas purF, purG, purC, guaA and guaB mutants were compromised in virulence in the mice model of infection. Moreover, purB mutant of Salmonella Typhimurium was also found to be avirulent ${ }^{14}$. Jelsbak et al. ${ }^{15}$ have reported that $\operatorname{purN}$ and $\operatorname{purT}$ are required for Salmonella Typhimurium growth in mice. purN and purT catalyse the same step in purine de novo biosynthesis and are considered to be redundant enzymes. Salmonella Typhimurium strains deficient in $\operatorname{purN}$ and purT grow as well as wildtype Salmonella Typhimurium in in vitro culture medium, indicating the redundancy of purN and purT. In the mice model of infection, $\operatorname{purN}$ and $p u r T$ individual mutants were attenuated in growth. Salmonella Typhimurium strains devoid of pyrE, a gene involved in de novo pyrimidine biosynthesis, was essential for infection in chicks but not in CBA/J mice ${ }^{16}$.

Commensal E. coli needs to synthesize purine and pyrimidine to colonize gnotobiotic mice intestine. $E$. coli mutants deficient in purC or pyrB were eliminated from mice intestine rapidly ${ }^{17}$. The purE mutation in Brucella melitensis $16 \mathrm{M}$ attenuates its growth in human monocytederived macrophages ${ }^{18}$. While screening the transposon mutant library of Brucella abortus strain RB51 for its intracellular growth ability in Hela cells, Truong et al. ${ }^{19}$ found that the purD and purF mutants were severely attenuated in intracellular growth. purD and purF mutants did not grow in in vitro complete medium also, whereas the supplementation of exogenous purine bases in the medium restored the growth of the mutants to wildtype level. Bacterial load in spleen of mice infected with purF and purD were significantly low as compared to 
bacterial load in the spleen of mice infected with wildtype $B$. abortus. The immunization of mice with purD and purF mutants confers protection when they are challenged with virulent bacteria ${ }^{19}$. Similar results of essentiality of purine biosynthesis for virulence of $B$. abortus strain 2308 in mice were reported. Mutation in de novo purine biosynthesis genes purE, purL and purD compromised the virulence in $\mathrm{BALB} / \mathrm{c}$ mice $^{20}$.

\section{Role of nucleotide salvage pathway in bacterial physiology and virulence}

The salvage pathway of nucleotide biosynthesis utilizes preformed nucleobases and nucleosides present in the internal milieu of cell or transported from external milieu into the cell via various nucleobase/nucleoside transporters to form nucleotides.

The salvage pathway bypasses the high-energy requiring de novo pathway and may be favoured under the conditions of low energy availability or rapid multiplication inside the host cell. Catalytic activity of adenylate kinase is essential for Streptococcus pneumoniae growth in vitro and in vivo ${ }^{21}$. Markaryan et al. ${ }^{22}$ have reported that Pseudomonas aeruginosa secretes adenylate kinase during infection. The secreted adenylate kinase acts as a virulence factor by converting ATP into a cytotoxic mixture of three nucleotides (AMP, ADP and ATP), which causes enhanced deaths in host macrophages. Yersinia pestis becomes avirulent in mice by introducing a mutation (P87S) in its adenylate kinase gene ${ }^{23}$. The role of nucleoside diphosphate kinase in bacterial physiology and virulence has been explicitly reviewed by $\mathrm{Yu}$ et $\mathrm{al}^{24}{ }^{24}$. It plays a crucial role in modulating virulence-associated phenotypes such as quorum sensing, type-III secretion, and virulence factor production. The secreted nucleoside diphosphate kinases bring about modulation in host responses ${ }^{24}$.

\section{Dependence of growth and virulence of fungal and parasitic protozoans on nucleotide biosynthesis}

Like bacterial pathogens, fungal pathogens also need to synthesize nucleotides by de novo pathway or scavenge nucleobase/nucleosides from the host to survive inside it and cause infection. The fungal pathogen Cryptococcus neoformans is commonly found in purine-rich bird guano and it has to undergo a drastic change in its nucleotide milieu when it infects a human host. Various genes of the purine biosynthesis pathway have been shown to be essential for growth and virulence of $C$. neoformans. The inosine monophosphate dehydrogenase (IMPDH) that converts inosine monophosphate into xanthosine monophosphate, which in turn is converted to guanosine monophosphate, is crucial for $C$. neoformans growth and virulence.
IMPDH-deleted mutant of C. neoformans exhibited reduced capsule and melanin (virulence factor) production as compared to wildtype and complemented strains. The ability of $C$. neoformans to cause infection in mice was abrogated with IMPDH gene deletion, and no fungi were found in the spleen and lung of mice after 7 days of infection $^{25}$. Ablation of GMP synthase which converts xanthosine monophosphate to guanosine monophosphate makes C. neoformans guanine auxotroph. The C. neoformans strain devoid of GMP synthase exhibits reduced production of virulence factors and compromised virulence in nematode as well as mice mode of infection ${ }^{26}$. AMP formation via the de novo pathway is fundamental for survival and virulence of $C$. neoformans, whereas AMP formation via the salvage pathway is redundant and is not required for its survival and virulence. Adenylosuccinate synthetase (AdSS) that catalyses the formation of adenylosuccinate (s-AMP) from IMP is required for virulence of $C$. neoformans in the mice model of infection. The $C$. neoformans mutant lacking the adss gene is an adenine auxotroph; however, the mutant is not impaired in the production of virulence factors implying that purine auxotrophy solely mediates the avirulent phenotype ${ }^{27}$. Adenylosuccinate lyase is a bifunctional enzyme involved in two chemical reactions in de novo purine biosynthesis. First, it catalyses the intermediary steps in the formation of IMP and thereafter it catalyses the formation of AMP from IMP. Thus, it affects both AMP and GMP production in the cell. The ability of neoformans to produce virulence factors like proteases, capsule and melanin pigment is compromised in the strain lacking adenylosuccinate lyase. In the murine model of infection, all the mice infected with the wildtype strain died within 25 days of infection, while the mice infected with adenylosuccinate lyase deficient strain survived and did not show any sign of infection till the end point of the experiment ${ }^{28}$.

Toxoplasma gondii, a protozoan parasite, is dependent on the pyrimidine de novo pathway for its pyrimidine requirement. Carbamoyl phosphate synthetase II, an enzyme of the pyrimidine de novo pathway is essential for $T$. gondii virulence in mice, and infection with $T$. gondii mutant lacking carbomyl phosphate synthetase II provides protective immunity to mice from subsequent $T$. gondii infection ${ }^{29}$. In another protozoan parasite, Trypanosoma brucei, GMP synthase is crucial for fulfiling the guanine requirement of parasite and deletion of GMP synthase restricts the growth of $T$. brucei bloodstream form in vitro. The exogenous supplementation of guanine at a concentration of $100 \mu \mathrm{M}$ rescued the growth defect of GMP synthase null mutant. The pharmacological inhibitor of GMP synthase inhibited the growth of the parasite. The infection of mice with wildtype parasite and GMP synthase null parasite demonstrated that the mutant parasite was unable to establish infection in mice with no detectable mutant parasites in the blood of mice post-30 days of infection ${ }^{30}$. 


\section{Summary}

The emergence of antibiotic-resistant bacterial and fungal pathogens calls for a need to develop novel therapeutic strategies. Drugs with new mechanisms of action are required urgently. In recent years, researchers have targeted essential central metabolic pathways of bacteria as potential drug targets. The drug targets include enzymes involved in the biosynthesis of nucleotides, amino acids and utilization of carbon sources. Essentiality of nucleotide biosynthetic pathway for pathogens during infection makes it an attractive molecular target. Some of the purine analogues are already found to be toxic to bacterial cell growth. Nucleobase and nucleoside analogues have been effectively used in the treatment of cancer and viruses, but they have not conventionally been considered as antibacterial agents, largely because of the success of other antimicrobial and toxicities related with nucleoside analogues. Although some of these enzymes involved in nucleotide biosynthesis are common between a pathogen and the human host, it may be possible to develop inhibitors with selective activity against the enzymes of pathogens by exploiting differences in their structural and biochemical characteristics.

1. Jensen, K. F., Dandanell, G., Hove-Jensen, B. and WillemoEs, M. Nucleotides, nucleosides, and nucleobases. EcoSal. Plus, 2008, 3.

2. Hopp, A. K., Gruter, P. and Hottiger, M. O., Regulation of glucose metabolism by NAD $(+)$ and ADP-ribosylation. Cells, 2019, 8 .

3. Zimmer, B. M., Barycki, J. J. and Simpson, M. A., Integration of sugar metabolism and proteoglycan synthesis by UDP-glucose dehydrogenase. J. Histochem. Cytochem., 2020, 22155420947500.

4. Gomelsky, M., Camp, c-di-GMP, c-di-AMP and now cGMP: bacteria use them all! Mol. Microbiol., 2011, 79, 562-565.

5. Turnbough Jr, C. L. and Switzer, R. L., Regulation of pyrimidine biosynthetic gene expression in bacteria: repression without repressors. Microbiol. Mol. Biol. Rev., 2008, 72, 266-300.

6. Samant, S., Lee, H., Ghassemi, M., Chen, J., Cook, J. L., Mankin, A. S. and Neyfakh, A. A., Nucleotide biosynthesis is critical for growth of bacteria in human blood. PLOS Pathog., 2008, 4, e37.

7. Connolly, J., Boldock, E., Prince, L. R., Renshaw, S. A., Whyte, M. K. and Foster, S. J., Identification of Staphylococcus aureus factors required for pathogenicity and growth in human blood. Infect. Immunol., 2017, 85.

8. Mei, J. M., Nourbakhsh, F., Ford, C. W. and Holden, D. W., Identification of Staphylococcus aureus virulence genes in a murine model of bacteraemia using signature-tagged mutagenesis. Mol. Microbiol., 1997, 26, 399-407.

9. Lan, L., Cheng, A., Dunman, P. M., Missiakas, D. and He, C., Golden pigment production and virulence gene expression are affected by metabolisms in Staphylococcus aureus. J. Bacteriol., 2010, 192, 3068-3077.

10. Li, L. et al., Role of purine biosynthesis in persistent methicillinresistant Staphylococcus aureus infection. J. Infect. Dis., 2018, 218, 1367-1377.

11. Kofoed, E. M. et al., De novo guanine biosynthesis but not the rioswitch-regulated purine salvage pathway is required for Staphylococcus aureus infection in vivo. J. Bacteriol., 2016, 198, 20012015.

12. Kriegeskorte, A. et al., Inactivation of thya in Staphylococcus aureus attenuates virulence and has a strong impact on metabolism and virulence gene expression. MBiol., 2014, 5, e01447-e01414.
13. Jenkins, A., Cote, C., Twenhafel, N., Merkel, T., Bozue, J. and Welkos, S., Role of purine biosynthesis in Bacillus anthracis pathogenesis and virulence. Infect. Immunol., 2011, 79, 153-166.

14. McFarland, W. C. and Stocker, B. A., Effect of different purine auxotrophic mutations on mouse-virulence of a VI-positive strain of Salmonella dublin and of two strains of Salmonella typhimurium. Microb. Pathog., 1987, 3, 129-141.

15. Jelsbak, L., Mortensen, M. I. B., Kilstrup, M. and Olsen, J. E., The in vitro redundant enzymes purN and purT are both essential for systemic infection of mice in Salmonella enterica serovar typhimurium. Infect. Immunol., 2016, 84, 2076-2085.

16. Yang, H. J., Bogomolnaya, L., McClelland, M. and AndrewsPolymenis, H., De novo pyrimidine synthesis is necessary for intestinal colonization of Salmonella Typhimurium in chicks. PLOS ONE, 2017, 12, e0183751.

17. Vogel-Scheel, J., Alpert, C., Engst, W., Loh, G. and Blaut, M., Requirement of purine and pyrimidine synthesis for colonization of the mouse intestine by Escherichia coli. Appl. Environ. Microbiol., 2010, 76, 5181-5187.

18. Drazek, E. S., Houng, H. S., Crawford, R. M., Hadfield, T. L., Hoover, D. L. and Warren, R. L., Deletion of purE attenuates Brucella melitensis $16 \mathrm{~m}$ for growth in human monocyte-derived macrophages. Infect. Immun., 1995, 63, 3297-3301.

19. Truong, Q. L., Cho, Y., Barate, A. K., Kim, S., Watarai, M. and Hahn, T. W., Mutation of purD and purF genes further attenuates Brucella abortus strain rb51. Microb. Pathog., 2015, 79, 1-7.

20. Alcantara, R. B., Read, R. D., Valderas, M. W., Brown, T. D. and Roop, R. M., Intact purine biosynthesis pathways are required for wild-type virulence of Brucella abortus 2308 in the balb/c mouse model. Infect. Immunol., 2004, 72, 4911-4917.

21. Thach, T. T., Luong, T. T., Lee, S. and Rhee, D. K., Adenylate kinase from Streptococcus pneumoniae is essential for growth through its catalytic activity. FEBS Open Biol., 2014, 4, 672-682.

22. Markaryan, A., Zaborina, O., Punj, V. and Chakrabarty, A. M., Adenylate kinase as a virulence factor of Pseudomonas aeruginosa. J. Bacteriol., 2001, 183, 3345-3352.

23. Munier-Lehmann, H., Chenal-Francisque, V., Ionescu, M., Chrisova, P., Foulon, J., Carniel, E. and Barzu, O., Relationship between bacterial virulence and nucleotide metabolism: a mutation in the adenylate kinase gene renders Versinia pestis avirulent. Biochem. J., 2003, 373, 515-522.

24. Yu, H., Rao, X. and Zhang, K., Nucleoside diphosphate kinase (NDK): a pleiotropic effector manipulating bacterial virulence and adaptive responses. Microbiol. Res., 2017, 205, 125-134.

25. Morrow, C. A. et al., De novo GTP biosynthesis is critical for virulence of the fungal pathogen Cryptococcus neoformans. PLOS Pathog., 2012, 8, e1002957.

26. Chitty, J. L. et al., Gmp synthase is required for virulence factor production and infection by Cryptococcus neoformans. J. Biol. Chem., 2017, 292, 3049-3059.

27. Blundell, R. D. et al., Disruption of de novo adenosine triphosphate (ATP) biosynthesis abolishes virulence in Cryptococcus neoformans. ACS Infect. Dis., 2016, 2, 651-663.

28. Chitty, J. L. et al., Cryptococcus neoformans ADS lyase is an enzyme essential for virulence whose crystal structure reveals features exploitable in antifungal drug design. J. Biol. Chem., 2017, 292, 11829-11839.

29. Fox, B. A. and Bzik, D. J., De novo pyrimidine biosynthesis is required for virulence of Toxoplasma gondii. Nature, 2002, 415, 926-929.

30. Li, Q., Leija, C., Rijo-Ferreira, F., Chen, J., Cestari, I., Stuart, K., Tu, B. P. and Phillips, M. A., Gmp synthase is essential for viability and infectivity of Trypanosoma brucei despite a redundant purine salvage pathway. Mol. Microbiol., 2015, 97, 1006-1020.

Received 4 July 2019; revised accepted 9 January 2021

doi: $10.18520 / \mathrm{cs} / \mathrm{v} 120 / \mathrm{i} 9 / 1458-1463$ 